\title{
KETEROBSERVASIAN SISTEM DESKRIPTOR DISKRIT LINIER
}

\author{
DIANA SYAFRIDA \\ Program Studi Matematika, \\ Fakultas Matematika dan Ilmu Pengetahuan Alam, Universitas Andalas, \\ Kampus UNAND Limau Manis Padang, Indonesia, \\ dianasyafrida.dsy@gmail.com
}

\begin{abstract}
Abstrak. Dalam penelitian ini akan dikaji keterobservasian sistem deskriptor diskrit linier yang regular bebas waktu dengan matriks $E$ adalah singular. Kajian ini dimulai dari mendekomposisi sistem sehingga dapat direduksi menjadi dua subsistem, yaitu slow subsystem dan fast subsystem. Selanjutnya, untuk menguji keterobservasian sistem akan digunakan kriteria keterobservasian yang menggunakan slow subsystem.
\end{abstract}

Kata Kunci: Sistem deskriptor diskrit linier, metode dekomposisi standar, keterobservasian sistem

\section{Pendahuluan}

Diberikan suatu sistem deskriptor diskrit linier sebagai berikut:

$$
\begin{aligned}
E \mathbf{x}(t+1) & =A \mathbf{x}(t)+B \mathbf{u}(t), \mathbf{x}(0)=\mathbf{x}_{0}, \\
\mathbf{y}(t) & =C \mathbf{x}(t),
\end{aligned}
$$

di mana $\mathbf{x}=\mathbf{x}(t) \in \mathbb{R}^{n}$ menyatakan vektor keadaan (state), $\mathbf{u}=\mathbf{u}(t) \in \mathbb{R}^{m}$ menyatakan vektor input dan $\mathbf{y}=\mathbf{y}(t) \in \mathbb{R}^{p}$ menyatakan vektor output. $E, A \in$ $\mathbb{R}^{n \times n}, B \in \mathbb{R}^{n \times m}, C \in \mathbb{R}^{p \times n}$, dan $t \in \mathbb{Z}_{+}$menyatakan waktu [5]. Jika matriks $E$ nonsingular, yaitu $\operatorname{det}(E) \neq 0$, berarti $E^{-1}$ ada, maka sistem (1.1) dapat ditulis menjadi

$$
\begin{aligned}
\mathbf{x}(t+1) & =\bar{A} \mathbf{x}(t)+\bar{B} \mathbf{u}(t), \\
\mathbf{y}(t) & =C \mathbf{x}(t),
\end{aligned}
$$

di mana $\bar{A}=E^{-1} A, \bar{B}=E^{-1} B$. Sistem (1.2) disebut juga sistem normal. Jika matriks $E$ singular, yaitu $\operatorname{det}(E)=0$, berarti $E^{-1}$ tidak ada, maka sistem (1.1) dapat didekomposisi dengan menunjukkan adanya matriks nonsingular $P, Q$ sedemikian sehingga sistem (1.1) dapat direduksi menjadi dua subsistem, yaitu

$$
\begin{aligned}
\mathbf{x}_{1}(t+1) & =A_{1} \mathbf{x}_{1}(t)+B_{1} \mathbf{u}(t) \\
\mathbf{y}_{1}(t) & =C_{1} \mathbf{x}_{1}(t), \operatorname{dan} \\
N \mathbf{x}_{2}(t+1) & =\mathbf{x}_{2}(t)+B_{2} \mathbf{u}(t) \\
\mathbf{y}_{2} & =C_{2} \mathbf{x}_{2}(t)
\end{aligned}
$$


di mana $\mathbf{x}_{1} \in \mathbb{R}^{n_{1}}, \mathbf{x}_{2} \in \mathbb{R}^{n_{2}}, A_{1} \in \mathbb{R}^{n_{1} \times n_{1}}, B_{1} \in \mathbb{R}^{n_{1} \times m}, B_{2} \in \mathbb{R}^{n_{2} \times m}, C_{1} \in \mathbb{R}^{p \times n_{1}}$, $C_{2} \in \mathbb{R}^{p \times n_{2}}, n_{1}=\operatorname{deg} \operatorname{det}(s E-A), n_{1}+n_{2}=n, \mathbf{y}=\mathbf{y}_{1}+\mathbf{y}_{2}, \operatorname{dan} N \in \mathbb{R}^{n_{2} \times n_{2}}$ adalah matriks nilpotent dengan indeks nilpotensi $h$. Sistem (1.3) disebut slow subsystem $\left(S_{1}\right)$ dan sistem (1.4) disebut fast subsystem $\left(S_{2}\right)$.

Dalam [2] dinyatakan bahwa sistem (1.1) dikatakan terobservasi, bila terdapat suatu waktu $t_{f}>0$ sedemikian sehingga jika $\mathbf{u}(t)$ dan $\mathbf{y}(t)$ diberikan pada $\left[0, t_{f}\right]$, maka $\mathbf{x}(0)=\mathbf{x}_{0}$ dapat ditentukan. Definisi ini juga berlaku untuk sistem normal. Dalam berbagai permasalahan, penggunaan definisi ini untuk menentukan apakah suatu sistem terobservasi atau tidak adalah sulit. Kriteria berikut dapat digunakan untuk menentukan keterobservasian dari sistem normal [2]. Sistem normal (1.2) adalah terobservasi jika dan hanya jika $\operatorname{rank}\left(\begin{array}{c}s I-\bar{A} \\ C\end{array}\right)=n, \forall s \in \mathbb{C}$, dengan $s$ berhingga.

\section{Solusi Sistem Deskriptor Diskrit Linier}

Sebelum menguji keterobservasian sistem deskriptor diskrit linier, terlebih dahulu perlu dilakukan dekomposisi terhadap sistem, seperti yang diberikan dalam teorema berikut.

Teorema 2.1. Sistem (1.1) adalah regular jika dan hanya jika terdapat matriks nonsingular $Q, P \in \mathbb{R}^{n \times n}$ sedemikian sehingga

$$
Q E P=\left(\begin{array}{cc}
I_{n_{1}} & O \\
O & N
\end{array}\right) \quad \text { dan } Q A P=\left(\begin{array}{cc}
A_{1} & O \\
O & I_{n_{2}}
\end{array}\right),
$$

di mana $A_{1} \in \mathbb{R}^{n_{1} \times n_{1}}, N \in \mathbb{R}^{n_{2} \times n_{2}}, n_{1}+n_{2}=n$, dan matriks $N$ adalah matriks nilpotent dengan indeks nilpotensi $h$.

Bukti. $(\Rightarrow)$ Misalkan sistem (1.1) regular. Maka $\operatorname{det}(s E-A) \neq 0$ untuk suatu $s \in \mathbb{R}$ sehingga $(s E-A)^{-1}$ ada. Misalkan

$$
\hat{E}=(s E-A)^{-1} E \text { dan } \hat{A}=(s E-A)^{-1} A .
$$

Maka

$$
\begin{aligned}
\hat{A} & =(s E-A)^{-1}(s E+A-s E), \\
& =-I+s(s E-A)^{-1} E, \\
& =s \hat{E}-I .
\end{aligned}
$$

Karena $E$ singular, maka $\hat{E}$ juga singular. Akibatnya, terdapat matriks nonsingular $T \in \mathbb{R}^{n \times n}$ sedemikian sehingga

$$
T^{-1} \hat{E} T=\left(\begin{array}{cc}
E_{1} & O \\
O & E_{2}
\end{array}\right),
$$

di mana $E_{1} \in \mathbb{R}^{n_{1} \times n_{1}}$ adalah nonsingular dan $E_{2} \in \mathbb{R}^{n_{2} \times n_{2}}$ adalah nilpotent. Akibatnya, $\left(E_{2} s-I\right)$ adalah nonsingular. Perhatikan kesamaan berikut

$$
(\alpha E-A)=(\alpha-s) E+(s E-A),
$$


untuk suatu $\alpha \in \mathbb{R}$. Kalikan kedua ruas (2.2) dengan $(s E-A)^{-1}$, diperoleh

$$
(s E-A)^{-1}(\alpha E-A)=(\alpha-s) T\left(\begin{array}{cc}
E_{1} & O \\
O & E_{2}
\end{array}\right) T^{-1}+I .
$$

Dapat dilihat bahwa (2.3) ekivalen dengan

$$
T^{-1}(s E-A)^{-1}(\alpha E-A) T=\left(\begin{array}{cc}
(\alpha-s) E_{1}+I_{n_{1}} & O \\
O & -\left(s E_{2}-I_{n_{2}}\right)+\alpha E_{2}
\end{array}\right) .
$$

Selanjutnya, kalikan kedua ruas (2.4) dengan

$$
\left(\begin{array}{cc}
E_{1}^{-1} & O \\
O & \left(s E_{2}-I_{n_{2}}\right)^{-1}
\end{array}\right)
$$

diperoleh

$$
\begin{aligned}
& \left(\begin{array}{cc}
E_{1}^{-1} & O \\
O & \left(s E_{2}-I_{n_{2}}\right)^{-1}
\end{array}\right) T^{-1}(s E-A)^{-1}(\alpha E-A) T \\
& =\alpha\left(\begin{array}{cc}
I_{n_{1}} & O \\
O & \left(s E_{2}-I_{n_{2}}\right)^{-1} E_{2}
\end{array}\right)-\left(\begin{array}{cc}
s I_{n_{1}}-E_{1}^{-1} & O \\
O & I_{n_{2}}
\end{array}\right) .
\end{aligned}
$$

Tulis

$$
Q=\left(\begin{array}{cc}
E_{1}^{-1} & O \\
O & \left(s E_{2}-I_{n_{2}}\right)^{-1}
\end{array}\right) T^{-1}(s E-A)^{-1} \text { dan } P=T .
$$

sehingga persamaan (2.5) dapat ditulis sebagai

$$
Q(\alpha E-A) P=\alpha\left(\begin{array}{cc}
I_{n_{1}} & O \\
O & N
\end{array}\right)-\left(\begin{array}{cc}
A_{1} & O \\
O & I_{n_{2}}
\end{array}\right),
$$

di mana $N=\left(s E_{2}-I_{n_{2}}\right)^{-1} E_{2}$ dan $A_{1}=s I_{n_{1}}-E_{1}^{-1}$. Jadi, terdapat $Q, P$ sehingga berlaku (2.1).

$(\Leftarrow)$ Misalkan terdapat matriks $Q, P \in \mathbb{R}$ sedemikian sehingga (2.1) berlaku. Misalkan $s \notin \sigma\left(A_{1}\right)$. Tanpa mengurangi keumuman, misalkan $s \in \mathbb{R}$. Maka $\operatorname{det}\left(s I_{n_{1}}-A_{1}\right) \neq 0$. Karena

$$
\begin{aligned}
\operatorname{det}\left(s N-I_{n_{2}}\right) & =\operatorname{det}\left(-\left(I_{n_{2}}-s N\right)\right), \text { dengan } s \neq 0, \\
& =(-1)^{n_{2}} s^{n_{2}}\left(\frac{1}{s}\right)^{n_{2}}, \\
& =(-1)^{n_{2}},
\end{aligned}
$$

maka

$$
\begin{aligned}
\operatorname{det}(s E-A) & =\operatorname{det}\left(s Q^{-1} Q E P P^{-1}-Q^{-1} Q A P P^{-1}\right) \\
& =\operatorname{det}\left(Q^{-1}\right) \operatorname{det}(s Q E P-Q A P) \operatorname{det}\left(P^{-1}\right) \\
& =\operatorname{det}\left(Q^{-1}\right) \operatorname{det}\left(s I_{n_{1}}-A_{1}\right)(-1)^{n_{2}} \operatorname{det}\left(P^{-1}\right) \\
& \neq 0 .
\end{aligned}
$$

sehingga sistem (1.1) regular. 
Berdasarkan Teorema 2.1 matriks $E, A \in \mathbb{R}^{n \times n}$ dapat didekomposisi sedemikian sehingga

$$
E=Q^{-1}\left(\begin{array}{cc}
I_{n_{1}} & O \\
O & N
\end{array}\right) P^{-1} \text { dan } A=Q^{-1}\left(\begin{array}{cc}
A_{1} & O \\
O & I_{n_{2}}
\end{array}\right) P^{-1}
$$

untuk suatu matriks nonsingular $P, Q \in \mathbb{R}^{n \times n}$.

Misalkan $\mathbf{x}(t)=P \mathbf{z}(t)$, untuk suatu $\mathbf{z}(t) \in \mathbb{R}^{n}$. Maka sistem (1.1) dapat ditulis menjadi

$$
\begin{aligned}
E P \mathbf{z}(t+1) & =A P \mathbf{z}(t)+B \mathbf{u}(t), \\
\mathbf{y}(t) & =C P \mathbf{z}(t) .
\end{aligned}
$$

Dengan mengalikan kedua ruas (2.7) dengan matriks nosingular $Q$, diperoleh

$$
\begin{aligned}
Q E P \mathbf{z}(t+1) & =Q A P \mathbf{z}(t)+Q B \mathbf{u}(t), \\
\mathbf{y}(t) & =C P \mathbf{z}(t) . \\
\left(\begin{array}{cc}
I_{n_{1}} & O \\
O & N
\end{array}\right)\left(\begin{array}{c}
\mathbf{z}_{1}(t+1) \\
\mathbf{z}_{2}(t+1)
\end{array}\right) & =\left(\begin{array}{cc}
A_{1} & O \\
O & I_{n_{2}}
\end{array}\right)\left(\begin{array}{c}
\mathbf{z}_{1}(t) \\
\mathbf{z}_{2}(t)
\end{array}\right)+\left(\begin{array}{c}
B_{1} \\
B_{2}
\end{array}\right) \mathbf{u}(t), \\
\mathbf{y}(t) & =\left(\begin{array}{ll}
C_{1} & C_{2}
\end{array}\right) \mathbf{z}(t),
\end{aligned}
$$

di mana $Q B=\left(\begin{array}{l}B_{1} \\ B_{2}\end{array}\right)$, dan $C P=\left(C_{1} C_{2}\right)$.

Dari sistem (2.10) dapat dilihat bahwa sistem (1.1) dapat direduksi menjadi dua subsistem, yaitu:

$$
\begin{aligned}
\mathbf{z}_{1}(t+1) & =A_{1} \mathbf{z}_{1}(t)+B_{1} \mathbf{u}(t), \\
\mathbf{y}_{1}(t) & =C_{1} \mathbf{z}_{1}(t), \operatorname{dan} \\
N \mathbf{z}_{2}(t+1) & =I_{n_{2}} \mathbf{z}_{2}(t)+B_{2} \mathbf{u}(t), \\
\mathbf{y}_{2}(t) & =C_{2} \mathbf{z}_{2}(t) .
\end{aligned}
$$

Solusi dari (2.11) adalah

$$
\mathbf{z}_{1}(t)=A_{1}^{t} \mathbf{z}_{1}(0)+\sum_{j=0}^{t-1} A_{1}^{t-j-1} B_{1} \mathbf{u}(j), t=0,1,2, \cdots,
$$

sedangkan solusi (2.12) adalah

$$
\mathbf{z}_{2}(t)=-\sum_{j=0}^{h-1} N^{j} B_{2} \boldsymbol{u}(t+j), t \in \mathbb{Z}_{+} .
$$

Sehingga solusi sistem (1.1) adalah

$$
\mathbf{x}(t)=P\left(\begin{array}{c}
A_{1}^{t} \mathbf{z}_{1}(0)+\sum_{j=0}^{t-1} A_{1}^{t-j-1} B_{1} \mathbf{u}(j) \\
-\sum_{j=0}^{h-1} N^{j} B_{2} \mathbf{u}(t+j)
\end{array}\right)
$$




\section{Keterobservasian Sistem Deskriptor Diskrit Linier}

Teorema berikut akan digunakan untuk menguji apakah sistem (1.1) terobservasi atau tidak.

Teorema 3.1. [4] Sistem (1.1) adalah terobservasi jika dan hanya jika sistem (2.11) adalah terobservasi.

Bukti. $\mathbf{z}_{2}(t)$ dapat ditentukan dari (2.14) untuk $N, B_{2}$, dan $\mathbf{u}(t)$. Sehingga sistem (1.1) adalah terobservasi jika dan hanya jika $\mathbf{z}_{1}(t)$ dapat ditentukan dari (2.11) dan $\mathbf{y}(t)-C_{2} \mathbf{z}_{2}(t)$. Jadi, sistem (1.1) adalah terobservasi jika dan hanya jika sistem (2.11) adalah terobservasi.

Teorema 3.2. [4] Pernyataan berikut adalah ekivalen.

(1) Sistem (1.1) adalah terobservasi,

(2) $\operatorname{Rank}\left(\begin{array}{c}s I_{n_{1}}-A_{1} \\ C_{1}\end{array}\right)=n_{1}, \forall s \in \mathbb{C}$ dengan $s$ berhingga,

(3) $\operatorname{Rank}\left(\begin{array}{c}s E-A \\ C\end{array}\right)=n, \forall s \in \mathbb{C}$ dengan s berhingga,

(4) $\operatorname{ker}\left(s I-A_{1}\right) \bigcap \operatorname{ker}\left(C_{1}\right)=\{\boldsymbol{O}\}, \forall s \in \mathbb{C}$ dengan $s$ berhingga,

(5) $\operatorname{ker}(s E-A) \bigcap \operatorname{ker}(C)=\{\boldsymbol{O}\}, \forall s \in \mathbb{C}$ dengan $s$ berhingga.

\section{Bukti.}

(1) $\Leftrightarrow(2)$ Jelas dari Bab I dan Teorema 3.1.

$(2) \Rightarrow(3)$ Misal $\operatorname{rank}\left(\begin{array}{c}s I_{n_{1}}-A_{1} \\ C_{1}\end{array}\right)=n_{1}, \forall s \in \mathbb{C}$ dengan $s$ berhingga. Akan ditunjukkan bahwa $\operatorname{rank}\left(\begin{array}{c}s E-A \\ C\end{array}\right)=n, \forall s \in \mathbb{C}$ dengan $s$ berhingga. Perhatikan bahwa

$$
\begin{aligned}
\operatorname{rank}\left(\begin{array}{c}
s E-A \\
C
\end{array}\right) & =\operatorname{rank}\left(\begin{array}{c}
s Q E P-Q A P \\
C P
\end{array}\right), \\
& =\operatorname{rank}\left(\begin{array}{cc}
s I_{n_{1}}-A_{1} & O \\
C_{1} & O \\
O & s N-I_{n_{2}}
\end{array}\right), \\
& =\operatorname{rank}\left(\begin{array}{c}
s I_{n_{1}}-A_{1} \\
C_{1}
\end{array}\right)+\operatorname{rank}\left(s N-I_{n_{2}}\right), \\
& =n_{1}+n_{2}=n .
\end{aligned}
$$


$(3) \Rightarrow(2)$ Perhatikan persamaan berikut.

$$
\begin{aligned}
& \operatorname{rank}\left(\begin{array}{c}
s I_{n_{1}}-A_{1} \\
C_{1}
\end{array}\right)+\operatorname{rank}\left(s N-I_{n_{2}}\right)=\operatorname{rank}\left(\begin{array}{cc}
s I_{n_{1}}-A_{1} & O \\
O & s N-I_{n_{2}} \\
C_{1} & C_{2}
\end{array}\right), \\
& =\operatorname{rank}\left(\begin{array}{c}
s Q E P-Q A P \\
C P
\end{array}\right) \text {, } \\
& =\operatorname{rank}\left(\begin{array}{c}
s E-A \\
C
\end{array}\right) \text {. }
\end{aligned}
$$

Jadi,

$$
\begin{aligned}
\operatorname{rank}\left(\begin{array}{c}
s I_{n_{1}}-A_{1} \\
C_{1}
\end{array}\right) & =\operatorname{rank}\left(\begin{array}{c}
s E-A \\
C
\end{array}\right)-\operatorname{rank}\left(s N-I_{n_{2}}\right) \\
& =n-n_{2}=n_{1} .
\end{aligned}
$$

$(3) \Leftrightarrow(5) \operatorname{Rank}\left(\begin{array}{c}s E-A \\ C\end{array}\right)=n, \forall s \in \mathbb{C}$ dengan $s$ berhingga jika dan hanya jika $\operatorname{ker}\left(\begin{array}{c}s E-A \\ C\end{array}\right)=\{\mathbf{0}\}$. Karena

$$
\operatorname{ker}\left(\begin{array}{c}
s E-A \\
C
\end{array}\right)=\{\mathbf{0}\}=\operatorname{ker}(s E-A) \bigcap \operatorname{ker}(C),
$$

maka rank $\left(\begin{array}{c}s E-A \\ C\end{array}\right)=n, \forall s \in \mathbb{C}$ dengan $s$ berhingga jika dan hanya jika $\operatorname{ker}(s E-A) \bigcap \operatorname{ker}(C)=\{\mathbf{0}\}$.

(2) $\Leftrightarrow(4)$ Dapat dibuktikan dengan mengikuti penalaran bukti (3) $\Leftrightarrow(5)$.

\section{Contoh}

Diberikan sistem deskriptor diskrit linier

$$
\begin{aligned}
E \mathbf{x}(t+1) & =A \mathbf{x}(t)+B \mathbf{u}(t), \\
\mathbf{y}(t) & =C \mathbf{x}(t) .
\end{aligned}
$$

di mana

$$
E=\left(\begin{array}{ccc}
0 & 1 & 0 \\
0 & -1 & 0 \\
0 & 0 & 1
\end{array}\right), A=\left(\begin{array}{lll}
1 & 0 & 0 \\
0 & 1 & 0 \\
0 & 1 & 0
\end{array}\right), B=\left(\begin{array}{ll}
1 & 0 \\
0 & 0 \\
0 & 1
\end{array}\right), C=\left(\begin{array}{ccc}
1 & 0 & 1 \\
1 & 1 & -1
\end{array}\right) .
$$

Akan diperiksa apakah sistem ini terobservasi atau tidak.

$$
\operatorname{det}(s E-A)=\operatorname{det}\left(\begin{array}{ccr}
-1 & s & 0 \\
0 & -s-1 & 0 \\
0 & -1 & s
\end{array}\right)=s^{2}+s .
$$

Pilih $s=1$, diperoleh

$$
\operatorname{det}\left(\begin{array}{ccc}
-1 & s & 0 \\
0 & -s-1 & 0 \\
0 & -1 & s
\end{array}\right)=2 \neq 0
$$


Selanjutnya,

$$
\hat{E}=\left(\begin{array}{ccc}
0 & -\frac{1}{2} & 0 \\
0 & \frac{1}{2} & 0 \\
0 & \frac{1}{2} & 1
\end{array}\right)
$$

Karena setiap matriks bujursangkar similar dengan suatu matriks Jordan, maka terdapat matriks nonsingular $T \in \mathbb{R}^{n \times n}$ sedemikian sehingga

$$
T^{-1} \hat{E} T=\left(\begin{array}{cc}
E_{1} & 0 \\
0 & E_{2}
\end{array}\right)=\left(\begin{array}{ccc}
\frac{1}{2} & 0 & 0 \\
0 & 1 & 0 \\
0 & 0 & 0
\end{array}\right)
$$

di mana $E_{1}=\left(\begin{array}{ll}\frac{1}{2} & 0 \\ 0 & 1\end{array}\right), E_{2}=0$, dan $T=\left(\begin{array}{ccc}-1 & 0 & 1 \\ 1 & 0 & 0 \\ -1 & 1 & 0\end{array}\right)$. Dengan menggunakan $(2.5)$ diperoleh

$$
Q=\left(\begin{array}{ccc}
0 & -1 & 0 \\
0 & -1 & 1 \\
1 & 1 & 0
\end{array}\right) \text { dan } P=T=\left(\begin{array}{ccc}
-1 & 0 & 1 \\
1 & 0 & 0 \\
-1 & 1 & 0
\end{array}\right)
$$

Kemudian,

$$
Q E P=\left(\begin{array}{lll}
1 & 0 & 0 \\
0 & 1 & 0 \\
0 & 0 & 0
\end{array}\right), Q A P=\left(\begin{array}{ccc}
-1 & 0 & 0 \\
0 & 0 & 0 \\
0 & 0 & 1
\end{array}\right), Q B=\left(\begin{array}{ll}
0 & 0 \\
0 & 1 \\
1 & 0
\end{array}\right)
$$

dan

$$
C P=\left(\begin{array}{ccc}
-2 & 1 & 1 \\
1 & -1 & 1
\end{array}\right)
$$

Dengan menggunakan (2.1) diperoleh

Kemudian

$$
A_{1}=\left(\begin{array}{cc}
-1 & 0 \\
0 & 0
\end{array}\right), I_{n_{2}}=1, N=0, I_{n_{1}}=\left(\begin{array}{ll}
1 & 0 \\
0 & 1
\end{array}\right) \text {. }
$$

$$
B_{1}=\left(\begin{array}{ll}
0 & 0 \\
0 & 1
\end{array}\right), B_{2}=\left(\begin{array}{ll}
1 & 0
\end{array}\right), C_{1}=\left(\begin{array}{cc}
-2 & 1 \\
1 & -1
\end{array}\right), C_{2}=\left(\begin{array}{l}
1 \\
1
\end{array}\right) .
$$

Berdasarkan Teorema 3.2,

$$
\operatorname{rank}\left(\begin{array}{c}
s I-A_{1} \\
C_{1}
\end{array}\right)=\operatorname{rank}\left(\begin{array}{cc}
2 & 0 \\
0 & 1 \\
-2 & 1 \\
1 & -1
\end{array}\right)=2=n_{1},
$$

sehingga sistem adalah terobservasi.

Jika digunakan bagian ke-3 dari Teorema 3.2, diperoleh

$$
\operatorname{rank}\left(\begin{array}{c}
s E-A \\
C
\end{array}\right)=\operatorname{rank}\left(\begin{array}{ccc}
-1 & 1 & 0 \\
0 & -2 & 0 \\
0 & -1 & 1 \\
1 & 0 & 1 \\
1 & 1 & 0
\end{array}\right)=3 .
$$


Jika digunakan bagian ke-4 dari Teorema 3.2, diperoleh

$$
\operatorname{ker}\left(s I-A_{1}\right) \bigcap \operatorname{ker}\left(C_{1}\right)=\operatorname{ker}\left(\begin{array}{c}
s I-A_{1} \\
C_{1}
\end{array}\right)=\operatorname{ker}\left(\begin{array}{cc}
2 & 0 \\
0 & 1 \\
-2 & 1 \\
1 & -1
\end{array}\right)=\{\mathbf{0}\} .
$$

Jika digunakan bagian ke-5 dari Teorema 3.2, diperoleh

$$
\operatorname{ker}(s E-A) \bigcap \operatorname{ker}(C)=\operatorname{ker}\left(\begin{array}{ccc}
-1 & 1 & 0 \\
0 & -2 & 0 \\
0 & -1 & 1 \\
1 & 0 & 1 \\
1 & 1 & 0
\end{array}\right)=\{\mathbf{0}\},
$$

sehingga sistem terobservasi. Jadi, sistem (4.1) terobservasi.

\section{Ucapan Terima kasih}

Penulis mengucapkan terima kasih kepada Bapak Dr. Muhafzan, Bapak Dr. Admi Nazra, Ibu Dr. Lyra Yulianti, Ibu Arrival Rince Putri, MT, M.Si yang telah memberikan masukan dan saran sehingga paper ini dapat diselesaikan dengan baik.

\section{Daftar Pustaka}

[1] Anton, H. 1991. Aljabar Linier Elementer, Edisi Kedelapan-Jilid 1. Erlangga, Jakarta

[2] Duan, G. R. 2010. Analysis and Design of Descriptor Linear System. Springer. New York

[3] Meyer, C. D. 2000. Matrix Analysis and Applied Linear Algebra. SIAM, New York

[4] Kaczorek, T. 1992. Linear Control Systems. Vol. 1. Research Studies Press Ltd. England

[5] Dai, Liyi. 1989. Singular Control Systems. Springer. New York 